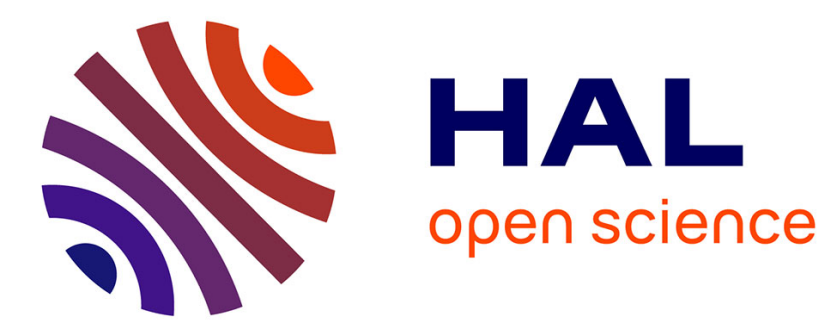

\title{
Shear Instabilities in Bidisperse Granular Flows
}

Axelle Amon, Renaud Delannay, Alexandre Valance

\section{To cite this version:}

Axelle Amon, Renaud Delannay, Alexandre Valance. Shear Instabilities in Bidisperse Granular Flows.

AIP Conference Proceedings, 2009, 1145, pp.583. 10.1063/1.3179994 . hal-00980621

\section{HAL Id: hal-00980621 \\ https://hal.science/hal-00980621}

Submitted on 18 Apr 2014

HAL is a multi-disciplinary open access archive for the deposit and dissemination of scientific research documents, whether they are published or not. The documents may come from teaching and research institutions in France or abroad, or from public or private research centers.
L'archive ouverte pluridisciplinaire HAL, est destinée au dépôt et à la diffusion de documents scientifiques de niveau recherche, publiés ou non, émanant des établissements d'enseignement et de recherche français ou étrangers, des laboratoires publics ou privés. 


\title{
Shear Instabilities in Bidisperse Granular Flows
}

\author{
A. Amon, R. Delannay and A. Valance \\ Institut de Physique de Rennes, UMR UR1-CNRS 6251, Université de Rennes 1, \\ Campus de Beaulieu, 35042 Rennes cedex, France
}

\begin{abstract}
We study experimentally the stability of the interface between two shearing granular flows. Both flows consist of monosized spherical glass beads but they differ in particle size. When running on an inclined rough plane, the two flows reach different stationary velocities. Hence, if their lateral sides get into contact, a shear zone appears at the interface. We show that in a limited region of the phase space (inclination angle/flow rate), the interface is unstable and develops a wavy pattern.
\end{abstract}

Keywords: granular flow ; shear instabilities

PACS: $45.70 . \mathrm{Mg}$

\section{INTRODUCTION}

Instabilities in granular flows have not been explored deeply yet [1]. In the case of shear instability (analogous to the Kelvin-Helmholtz instability in hydrodynamics), first experimental observations were reported by Goldfarb et al. [2]. However, such kind of instability is not fully characterized and the understanding of its mechanism is still open.

Goldfarb et al. [2] studied shear flows on an inclined chute. Two hoppers with different apertures allowed to obtain two flows with different rates. They used for both flows the same type of irregular sand, sieved or not. The rigid plane at the bottom of the channel was smooth and consequently the flows were accelerated along the chute. They observed instabilities at the interface between the two shearing flows only at small angles of inclination. When using spherical glass beads instead of sand, they did not observe any instability. The authors proposed a dimensionless number to characterize the competition between elongation due to acceleration and shear. They argued from their experiments that instabilities are favored when there is no elongation.

These observations motivated us to design a new experiment allowing to obtain a shear between two steady granular flows. In order to achieve such a configuration, we took advantage of the properties of monodisperse granular flows on bumpy inclined plane $[3,4]$. We created two granular streams of same height but differing in grain sizes. Experiencing a differential basal friction, they flow at different velocity producing a shear zone at the surface of contact. We investigated the behavior of the interface between these two streams according to the flow height and chute inclination. We showed in particular that the interface becomes unstable in a limited region of the phase space.

\section{EXPERIMENTAL SETUP}

Figure 1 shows a sketch of our experimental setup. The side walls of the chute are made of plexiglass. Two different chute configurations were used: a wide chute of $28.5 \mathrm{~cm}$ width and a narrow one of $13.2 \mathrm{~cm}$ width. The chute is $130 \mathrm{~cm}$ long and the base consists of $500 \mu \mathrm{m}$ spherical beads glued on a rigid plane. At the upper end of the chute, two independent reservoirs with variable outlets allow to trigger two granular streams. For our flow experiment, we used two kinds of spherical glass beads : white fine beads of $250 \mu \mathrm{m}$ diameter and black large ones of $500 \mu \mathrm{m}$ diameter.

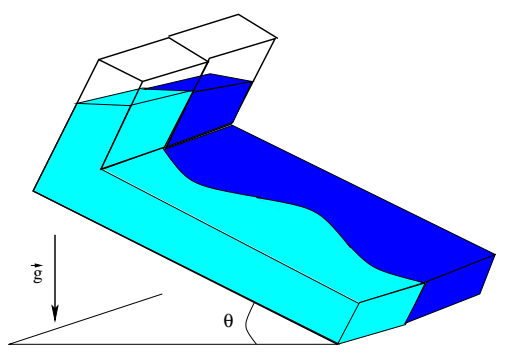

FIGURE 1. Sketch of the experimental set-up. $\theta$ is the inclination of the chute with respect to the horizontal and $\vec{g}$ is the acceleration of gravity.

Control parameters are the inclination angle of the plane with horizontal, $\theta$, controlled with a precision of $0.1^{\circ}$, and the thickness of the flowing layer, $h$, tuned with the outlet of the reservoirs. Two laser sheets hit the flow surface with small incident angles at two different positions along the chute. The displacement of their traces on the flow surface gives a measurement of the thickness of the flowing layer. A camera is placed above the flow to record the temporal evolution of the interface between the two granular streams. 


\section{FLOW FEATURES}

We first recall briefly the different regimes observed with monodisperse granular assemblies flowing on rough inclines [4]. For a given angle $\theta$ of inclination, granular layers thiner than a minimum thickness, $h_{\text {stop }}(\theta)$, comes into arrest. For thicknesses greater than $h_{\text {stop }}$, flows can be sustained: they are steady and fully developed for moderate inclination angle and thickness, but become accelerated for larger values of these parameters [4].

The ratio between the diameter of the flowing beads and that of the glued beads determines the effective friction experienced by the flow and consequently controls the flow velocity when steady uniform flow is obtained. For a given bumpiness of the plane, there exists a critical bead diameter for which the flow velocity is minimum, that is when the diameter of the flowing beads is approximatively half of the diameter of the glued beads [5].

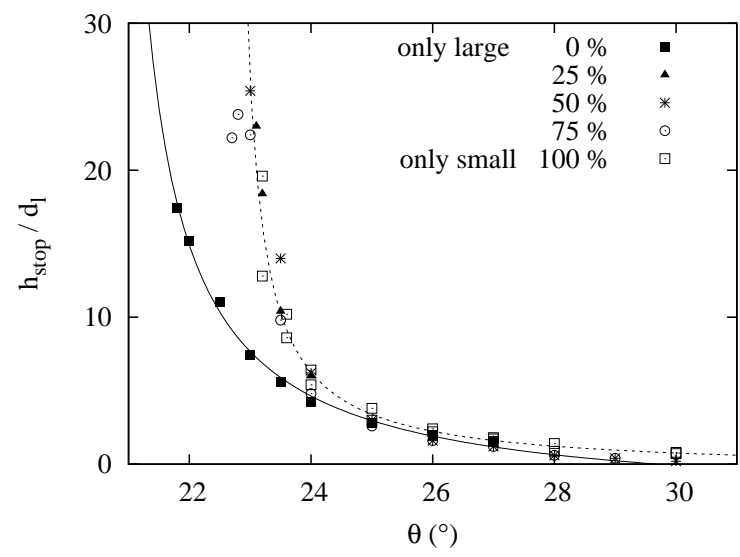

FIGURE 2. $h_{\text {stop }} / d_{l}$ versus $\theta$ for different mixtures of different proportions of fine and large grains. The solid and dashed lines correspond to best fits using eq. (1).

We determined the curves $h_{\text {stop }}(\theta)$ for monodisperse flows and mixtures using the two grain species. At a given fixed angle, we released the material placed in the reservoirs and waited for the flow to stop. The thickness of the remaining layer is $h_{\text {stop }}$. Figure 2 shows the data obtained for the wide chute. Note that $h_{\text {stop }}$ has been normalized by the diameter of the large beads, $d_{l}=500$ $\mu \mathrm{m}$. Data corresponding to monodisperse flows can be well approximated by the following phenomenological law $[3,4]$ :

$$
\frac{h_{\text {stop }}(\theta)}{d}=\gamma \frac{\tan \theta_{2}-\tan \theta}{\tan \theta-\tan \theta_{1}}
$$

where $d$ is the diameter of the flowing beads, and $\gamma, \theta_{1}$, $\theta_{2}$ are fit parameters whose values are given in table 1 for both bead diameters and chute widths. For a given grain size, the values obtained are approximately the same for both chute widths. Nevertheless, we noted a
TABLE 1. Fit parameters.

\begin{tabular}{c|l|lll|l}
\hline$d$ & channel & $\gamma$ & $\theta_{1}$ & $\theta_{2}$ & $\beta$ \\
\hline \multirow{2}{*}{$250 \mu \mathrm{m}$} & wide & .8 & $23^{\circ}$ & $42^{\circ}$ & .20 \\
& narrow & .8 & $22^{\circ}$ & $39^{\circ}$ & .31 \\
\hline \multirow{2}{*}{$500 \mu \mathrm{m}$} & wide & 2.6 & $21^{\circ}$ & $30^{\circ}$ & .13 \\
& narrow & 2.5 & $19^{\circ}$ & $28^{\circ}$ & .11 \\
\hline
\end{tabular}

variability along the plane, with a systematic tendency for $h_{\text {stop }}$ to decrease downstream along the slope. For a given inclination angle, $h_{\text {stop }}$ is higher for the fine beads than for the large ones. This is in agreement with the fact that $250 \mu \mathrm{m}$ beads experience a larger effective friction on a bumpy plane made with $500 \mu \mathrm{m}$ glued beads than the $500 \mu \mathrm{m}$ particles [5]. Data corresponding to mixtures collapse on the $h_{\text {stop }}$ curve obtained for the fine beads. We observed that the deposit obtained with mixtures is almost exclusively composed of fine grains. This is in agreement with the known behavior of polydisperse flows [6] : segregation causes the large grains to move towards the front and the surface of the flow, and when the flow stops, the remaining layer is mostly composed of fine grains.

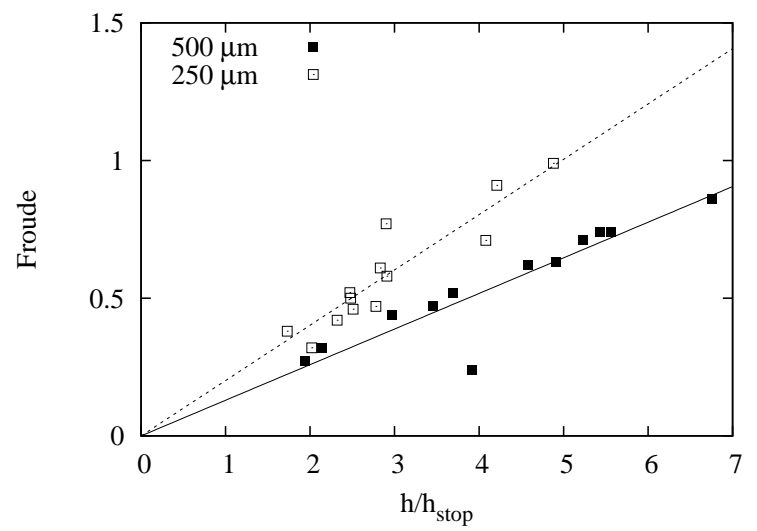

FIGURE 3. Froude number $v / \sqrt{g h}$ as a function of $h / h_{\text {stop }}$ for monodisperse flows. Filled symbols : $500 \mu \mathrm{m}$ beads; open symbols : $250 \mu \mathrm{m}$ beads. Solid and dotted lines are linear fits.

For steady flows of velocity $v$, the Froude number $v / \sqrt{g h}$ is expected to vary as [3] :

$$
\frac{v}{\sqrt{g h}}=\alpha+\beta \frac{h}{h_{\text {stop }}}
$$

where $\alpha$ and $\beta$ are parameters which depend on material properties of the beads but not on the grain diameter. For glass beads flowing over a rough plane made of glass beads, $\alpha=0$ and $\beta=0.136$ [4], whatever the bead diameter. Figure 3 shows data obtained with the wide chute. The slopes deduced from a linear fit are shown in the last column of table 1 . Taking into account 
the dispersion of the measurements, the slope values obtained for the $500 \mu \mathrm{m}$ beads fairly agree with the expected value. For the fine beads, there is a deviation from the expected value. Besides, the values of the slope obtained for both chute widths are different. We checked the granulometry of our beads and found that the fine beads have a much larger size distribution than the large ones. This could explain the discrepancy observed for large and small glass beads.

By using the difference of friction experienced by two types of beads on a bumpy plane, we can obtain for a given chute inclination steady flows with same height but different velocity. It is then possible to produce a shear between two granular streams running side by side on the same bumpy plane. In the next section, we present succinctly the behavior of the contact interface between both streams.

\section{RESULTS}

Figure 4 shows a snapshot of unstable shear flows : the interface between the black fine grains and the white large ones develops a wavy pattern. Analysis of successive images of the flow allows to extract the interface position and to characterize its temporal evolution. The wavelength of the pattern typically ranges from 10 to 25 $\mathrm{cm}$ and the frequency from 5 to $10 \mathrm{~Hz}$.

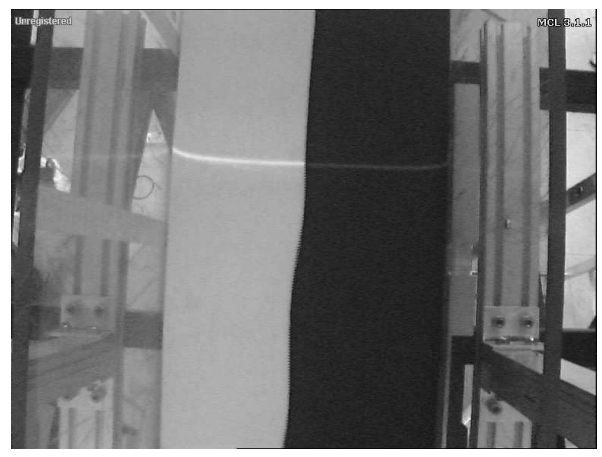

FIGURE 4. Top view of the two streams.

The instability of the interface is not observed in the whole parameter space. Figure 5 shows the parameter values for which flows were carried out. We display data for large (fig. 5(a)) and narrow (fig. 5(b)) chute widths. Filled symbols correspond to parameters for which instability of the interface was observed, while open symbols are associated with stable flows. The curves $h_{\text {stop }}(\theta)$ for fine and large grains are presented as well.

One can note that for small values of $h$, interface instability is rarely observed. A natural question arises: what is the criterion for the instability? As the flow rule is different for both streams (see eq. 2 and table 1), it follows that the velocity difference $v_{l}-v_{s}$ between the
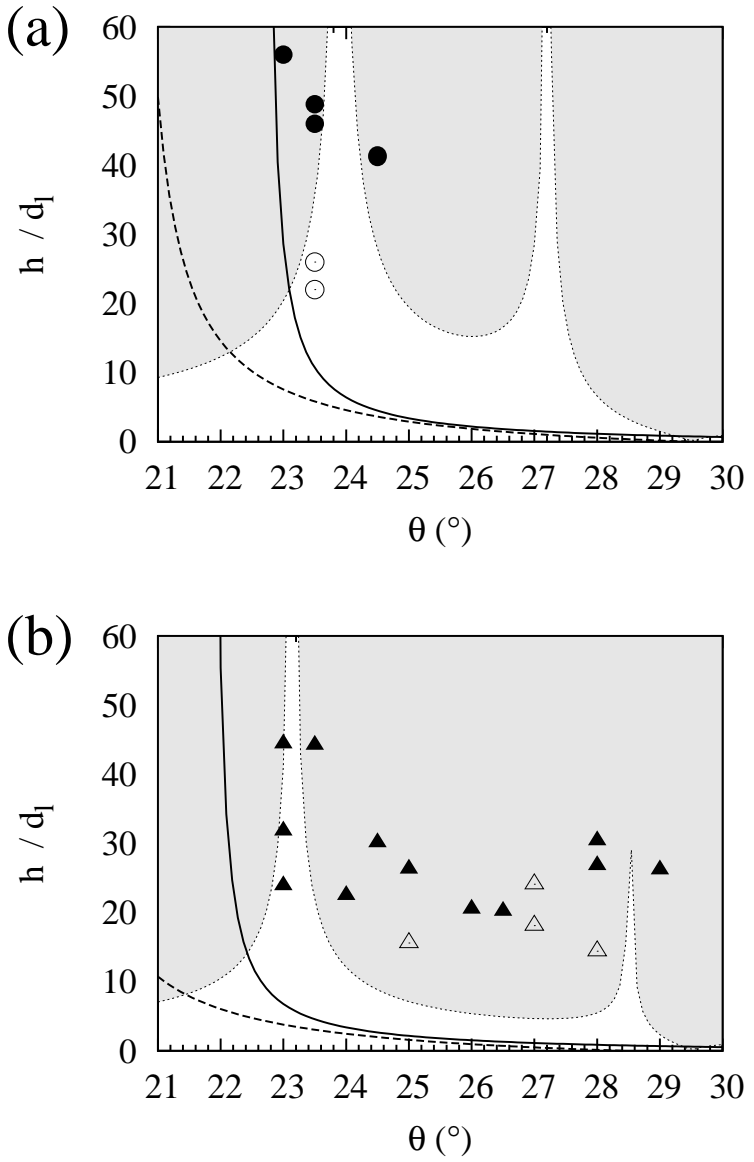

FIGURE 5. Phase diagram in the parameter space $\left(h / d_{l}, \boldsymbol{\theta}\right)$ for both chute configurations: wide (a) and narrow (b) chute. Filled symbols: unstable flow; open symbols: stables flows. Solid and dotted lines stand for the curves $h_{\text {stop }}(\theta)$ respectively for fine and large grains. The domains in grey correspond to regions where the velocity difference $\left|v_{l}-v_{s}\right|$ is greater than $\sqrt{g h}$.

two flows can vanish and change sign according to the values of the control parameters $h$ and $\theta$. In figure 5, we colored in grey the region for which $\left|v_{l}-v_{s}\right|>\sqrt{g h}$. Experimental data suggest that a criterion based on the velocity difference may be plausible.

\section{CONCLUSION}

In this article, we present a simple granular experiment where two steady streams of grains experience a shear at their surface of contact. Each stream is composed of monosized spherical glass beads. Particles from the two streams differ only in size. We show that the interface between the two flows can become unstable and develop a wavy pattern. Contrary to experiments of Goldfarb 
et al., the instability is observed with regular spherical particles. In addition, we provided a phase diagram in the parameter space $(h, \theta)$ identifying the stable and unstable regions of the flow, and proposed a criterion for the instability onset based on the difference in flow velocity.

\section{ACKNOWLEDGMENTS}

We are grateful to several students who contributed to this work : R.M Guillermic, I. Hussein and S. Pasumarthy. We also thank A. Faisant for his advices concerning the design of the experimental set-up, S. Bourles and P. Chasle for technical assistance.

\section{REFERENCES}

1. I. S. Aranson and L. S. Tsimring, "Patterns and collective behavior in granular media : Theoretical concepts", Rev. Mod. Phys. 78, 641 (2006).

2. D. J. Goldfarb, B. J. Glasser and T. Shinbrot,"Shear instabilities in granular flows", Nature 415, 302 (2002).

3. GDR MiDi, "On dense granular flows", Eur. Phys. J. E 14, 341 (2004).

4. O. Pouliquen, "Scaling laws in granular flows down rough inclined planes", Phys. Fluids 11, 542 (1999).

5. C. Goujon, N. Thomas, and B. Dalloz-Dubrujeaud,

"Monodisperse dry granular flows on inclined planes: Role of roughness", Eur. Phys. J. E 11, 147 (2003).

6. C. Goujon, B. Dalloz-Dubrujeaud, and N. Thomas, "Bidisperse granular avalanches on inclined planes : A rich varity of behaviors", Eur. Phys. J. E 23, 199 (2007). 